\title{
Myths and misconceptions about intrauterine contraception among women seeking termination of pregnancy
}

\author{
Lucy Michie, ${ }^{1}$ Sharon T Cameron, ${ }^{2}$ Anna Glasier, ${ }^{3}$ Kaye Wellings, ${ }^{4}$ \\ Joanna Loudon ${ }^{5}$
}

For numbered affiliations see end of article.

\section{Correspondence to}

Dr Lucy Michie, Department of Reproductive and Developmental Sciences, University of Edinburgh, Edinburgh and Chalmers Sexual and Reproductive Health Centre, 51 Little France Crescent, Edinburgh EH16 5SU, UK; Lucy.Michie@ed.ac.uk

Received 21 September 2012 Revised 22 January 2013 Accepted 6 March 2013 Published Online First 24 May 2013
To cite: Michie $\mathrm{L}$, Cameron ST, Glasier A, et al. J Fam Plann Reprod Health Care 2014;40:36-40.

\begin{abstract}
Background Immediate initiation of an intrauterine device (IUD) or intrauterine system (IUS) following termination of pregnancy (TOP) is associated with a significant reduction in the risk of another TOP. In spite of its high efficacy, uptake of intrauterine contraception in the UK is low. Myths and misconceptions about the method may contribute to the low uptake.
\end{abstract} Study design Anonymous, self-administered questionnaire among women requesting a TOP in a hospital abortion service in Scotland, UK. Methods Misconceptions about intrauterine contraception were extracted from an online social networking and micro-blogging service, and from existing research to develop a questionnaire containing 12 negative statements about intrauterine contraception. Respondents indicated their level of agreement with each statement.

Results A total of 106/125 (85\%) women requesting a TOP completed the questionnaire. The two commonest negative statements that respondents agreed with were that the IUD/IUS 'Is painful to have inserted' ( $n=36 ; 34 \%)$ and that 'It can move around inside your body' $(n=25 ; 23.6 \%)$. The range of women who neither agreed nor disagreed with negative statements was 26.4-56.0\%. Twenty-seven (25\%) women indicated that the IUD/IUS was their planned method of post-TOP contraception.

Conclusions Although myths about intrauterine contraception persist among a small proportion of women requesting a TOP, lack of knowledge about the method is also evident. The consultation prior to TOP is an important opportunity to provide accurate and quality information to women about the IUD/IUS that may serve to increase uptake and prevent repeat abortions.

\section{Key message points}

Only a minority of women requesting a termination of pregnancy (TOP) hold misconceptions about intrauterine contraception, although knowledge of the method is lacking.

- The consultation prior to TOP is an important opportunity to provide accurate and quality information to women about intrauterine contraception that may serve to increase uptake of this method and protect women from a further TOP.

\section{INTRODUCTION}

The use of intrauterine contraception, either as an intrauterine device (IUD) or hormone-releasing intrauterine system (IUS), varies significantly across the world. Worldwide use was most recently estimated to be $14 \%$, although this rises in some countries with rates as high as 37\% in Eastern Asia. ${ }^{2}$ The UK, however, has much lower rates of use of intrauterine contraception (6\% using an IUD and 2\% using an IUS in 2010). ${ }^{3}$ Intrauterine contraception is considerably less popular in the UK than either oral contraception or condoms. ${ }^{3}$ A systematic review of the literature regarding contraceptive efficacy found the IUS to be as effective as female sterilisation, and the IUD was rated second to the IUS for effectiveness. ${ }^{4}$

In 2011, the rate of abortion per 1000 women aged $15-44$ years was 12.0 in Scotland and 17.5 in England and Wales. ${ }^{5}{ }^{6}$ National guidance from the UK 
recommends that increasing uptake of intrauterine contraception has the potential to reduce the number of termination of pregnancies (TOP). ${ }^{7}$ There is also global evidence that immediate initiation of intrauterine contraception at the time of TOP is associated with a significant reduction in the likelihood of subsequent TOP ${ }^{8-10}$ In 2011, 29\% of women having an abortion in Scotland and 35\% in England and Wales had at least one previous TOP. ${ }^{6}$ Increased uptake of intrauterine contraception among women having a TOP could therefore play an important role in reducing this repeat abortion rate. Unfortunately, myths and misconceptions about intrauterine contraception may account for the low uptake of this method in the UK. ${ }^{11} 12$

In order to determine what proportion of women seeking TOP hold misconceptions about the IUD/IUS we conducted a survey among women requesting TOP at a hospital abortion service at the Royal Infirmary of Edinburgh (RIE), Edinburgh, Scotland, UK. The RIE is the main provider (80\%) of abortion services in Lothian (Edinburgh and surrounding area). In 2011, 2416 induced abortions were conducted in Lothian. ${ }^{5}$ The purpose of the study was to provide information to help guide health professionals in developing effective educational strategies that may increase positive attitudes towards intrauterine contraception, so that more women may consider this as a method of ongoing contraception after a TOP.

\section{METHODS}

In order to help to develop a short questionnaire for women to complete regarding their beliefs about intrauterine contraception, two separate sources were used to identify common misconceptions women may have. First, statements about intrauterine contraceptives were taken from unpublished transcripts of interviews undertaken with young people aged 13-21 years during 2000-2004 as part of an evaluation of a national teenage pregnancy strategy. ${ }^{13}$ Second, we extracted negative statements about the IUD/IUS from an online social networking and micro-blogging service (Twitter) by conducting two searches 10 days apart in December 2011. The search terms used were 'IUD', 'intrauterine device', 'IUS', 'mirena', 'coil' and 'paragard'. Statements were identified that discussed the IUD, although those that used the term in an unrelated meaning were not included. By reviewing these sources common themes were identified regarding women's views towards, and concerns about, the IUD/ IUS. This allowed us to create a questionnaire comprising a short introductory paragraph followed by 12 negative statements about the IUD/IUS. During January and February 2012, women attending the RIE clinics requesting a TOP were given the questionnaire by one of the clinic nurses and invited to complete it and place it in an opaque sealed envelope in a collection box. The questionnaires were completed by women prior to either ultrasound scan or consultation with medical staff in the clinic, and they therefore were not aware of what method of TOP they could have (if at all), and had not discussed contraception with any medical staff in the clinic at that point. The questionnaire was anonymous and self-completed and required a response to each statement on a five-point Likert scale ranging from 'strongly agree' to 'strongly disagree'. Further questions sought demographic information including age, postcode area of residence (used to obtain a Carstairs deprivation category score $)^{14}$ and previous and intended contraceptive use.

\section{Statistics}

All the data were coded and entered onto a database using Microsoft Excel ${ }^{\mathrm{TM}}$. Data were entered into the database by a research nurse and the data were checked and coded by one of the authors (LM). Responses to each statement were combined such that 'strongly agree' and 'somewhat agree' were grouped as 'agree', while 'strongly disagree' and 'somewhat disagree' were grouped as 'disagree'. The remaining group of responses were 'neither agree nor disagree'. Data analysis was performed using IBM Statistical Package for Social Sciences (SPSS) software V.18 (IBM Corporation, New York, NY, USA). Demographic data were obtained including means and standard deviations. To allow statistical comparison between age groups, four age groups were defined: 0-19, 20-24, 25-34 and 35+ years. Comparisons were made using Fisher's exact test as counts within the individual cells of the contingency table fell below 5. Statistical significance was deemed to be $p<0.05$.

\section{Ethical approval}

The questionnaire was reviewed by the chair of a local research ethics committee who confirmed that ethical approval was not required for this study.

\section{RESULTS}

A total of 106 completed questionnaires were obtained from 125 distributed (an 85\% response rate). The age of respondents ranged from 15 to 42 years and their demographics are shown in Table 1 . Seventy-eight per cent of women $(n=83)$ had used more than one method of contraception previously, and eight $(8 \%)$ women had previously used an IUD or IUS in the past (Table 1). Regarding future planned use of contraception, three (3\%) women intended to use no contraception and two $(2 \%)$ were uncertain. Of those respondents $(n=101)$ who were intending to use contraception, the methods planned included oral contraceptive pill $(n=33 ; 31 \%)$, IUD or IUS $(n=27 ; 25 \%)$, progestogen-only implant $(n=26 ; 24 \%)$, barrier methods $(n=26,24 \%)$, progestogen-only injectable $(n=13 ; 12 \%)$, combined hormonal contraceptive patch $(n=3,3 \%)$ and sterilisation $(n=2,2 \%)$. Parous women were significantly more likely to indicate that they planned to use an IUD/IUS 
Table 1 Demographics of the questionnaire respondents $(n=106)$

\begin{tabular}{lc}
\hline Demographic & {$[\boldsymbol{n}(\%)]$} \\
\hline Age (years) & $25(6.4)$ \\
Mean (SD) & $15-42$ \\
Range & \\
Deprivation Category score* & $15(14.2)$ \\
1-2 Affluent & $77(72.6)$ \\
3-5 Moderate & $14(13.2)$ \\
6-7 Deprived & \\
Parity & $57(53.8)$ \\
Nulliparous & $49(46.2)$ \\
Parous & $36(34.0)$ \\
Previous abortion & \\
Previous methods of contraception ever used & $98(92)$ \\
Condom & $74(74)$ \\
Oral contraceptive pill & $16(15)$ \\
Progestogen-only implant & $12(11)$ \\
Progestogen-only injectable & $8(8)$ \\
Intrauterine device/system & $5(3)$ \\
Combined hormonal patch & $4(4)$ \\
None &
\end{tabular}

${ }^{*}$ Deprivation Category is a marker of deprivation in Scotland based upon postcode area of residence.

$\mathrm{SD}$, standard deviation.

for future contraception compared to nulliparous women $(p=0.009)$. Women who had previously had a TOP were also significantly more likely to choose the IUD/IUS as a future method when compared to women with no history of TOP $(p=0.039)$. Women who had used an IUD/IUS as a contraceptive method previously were significantly more likely to choose this as future contraception $(p=0.003)$.

The 12 statements presented to the women and the responses to each statement are shown in Table 2 . Agreement with negative statements ranged from 2.8\% to $34.0 \%$. The range in percentage of women who neither agreed nor disagreed with each negative statement was $26.4-56.0 \%$. The statements that most women agreed with were that 'It is painful to have inserted' (34.0\%) and that 'It can move around inside your body' (23.6\%). Responses were compared between women who had previously used an IUD or IUS and those who had never used these methods. Women who had used previously used intrauterine contraception were significantly more likely to disagree with Statement 5 ('There is a good chance it can make you infertile') than those who had not $(p=0.03)$. There was no significant difference in responses between these groups for all other statements. Women aged 19 years and under $(n=24)$ were significantly more likely to agree with Statement 1 ('It is painful to have inserted') compared to women in any other age group ( $p=0.037)$. There were no significant associations between any demographic factors tested (i.e. age group, deprivation category score, reproductive history) and agreement with any other statements.

\section{DISCUSSION}

Our study showed that only a small percentage of women requesting a TOP agreed with the negative statements about intrauterine contraception, suggesting that only a minority of these women held major misconceptions about this method. Our study did, however, show that approximately one-third of women 'neither agreed nor disagreed' with the statements, which may suggest a lack of knowledge about intrauterine contraception amongst this group. Thus the TOP consultation does offer a good opportunity to provide information about this most effective method of contraception that has been shown to reduce the risk of a subsequent TOP ${ }^{8-10}$ Some health professionals may worry that women requesting a TOP may not wish to discuss contraception at this

Table 2 Response to negative statements about intrauterine contraception

\begin{tabular}{|c|c|c|c|}
\hline \multirow[b]{2}{*}{ Statement } & \multicolumn{3}{|c|}{ Response $[n(\%)]$} \\
\hline & Agree & $\begin{array}{l}\text { Neither agree } \\
\text { nor disagree }\end{array}$ & Disagree \\
\hline 1 It is painful to have inserted & $36(34.0)$ & $59(56.0)$ & $11(10.0)$ \\
\hline 2 It is only suitable for women who have had children & $8(7.5)$ & $40(37.8)$ & $58(54.7)$ \\
\hline 3 It is not suitable if you have had more than three children & $3(2.8)$ & $53(50.0)$ & $50(47.2)$ \\
\hline 4 Can only be used in older women & $4(3.8)$ & $38(35.8)$ & $64(60.4)$ \\
\hline 5 There is a good chance it can make you infertile & $3(2.8)$ & $45(42.5)$ & $58(54.7)$ \\
\hline 6 There is a good chance it can damage the womb & $17(16.0)$ & $36(34.0)$ & $53(50.0)$ \\
\hline 7 There is a good chance it can damage the ovaries & $6(5.7)$ & $44(41.5)$ & $56(52.8)$ \\
\hline 8 It can rust inside you & $8(7.5)$ & $36(34.0)$ & $62(58.5)$ \\
\hline 9 It can move around inside your body & $25(23.6)$ & $28(26.4)$ & $53(50.0)$ \\
\hline 10 There is a high chance it might fall out & $16(15.1)$ & $40(37.7)$ & $50(47.2)$ \\
\hline 11 It can get stuck on the baby's head if you become pregnant & $6(5.6)$ & $36(34.0)$ & $64(60.4)$ \\
\hline 12 It is a breeding ground for infection & $17(16.0)$ & $47(44.4)$ & $42(39.6)$ \\
\hline
\end{tabular}


time, or that they may feel under pressure to accept contraception in order to obtain agreement to have an abortion. However, there is good evidence that women value the opportunity to discuss contraception at this visit and do no feel coerced into accepting a method of contraception. ${ }^{15}$ Although the consultations to discuss TOP may be lengthy and the time available to discuss contraception is short, there is evidence from the USA that even brief ( 3 minutes) oral educational interventions about the IUD/IUS can improve knowledge and positivity about this method. ${ }^{16-18}$ Furthermore, women seeking a TOP find that information about contraception imparted from viewing a digital video disk (DVD), rather than a face-to-face consultation with a health professional, is highly acceptable at this time. ${ }^{15}$

In our study the most common misconceptions about intrauterine contraception that women agreed with were that the IUD/IUS is painful to have inserted and that it can move around inside your body. This may indicate that health professionals need to concentrate on providing accurate information and reassurance to women about these issues. In particular, oral analgesia or local anaesthesia for insertion can be discussed with women, as is recommended by the Faculty of Sexual \& Reproductive Healthcare guidance. ${ }^{19}$ Women can also be reassured that the likelihood of an IUD/IUS perforating the uterus is rare. ${ }^{19}$

The demographic characteristics of women participating in our survey were similar to that of previous studies of women attending for TOP in our region. ${ }^{10}$ In addition, our finding of $8 \%$ of women having previously used an IUD/IUS is in keeping with rates of uptake of intrauterine contraception in the UK. ${ }^{3}$ More surprising was the finding that $25 \%$ of respondents were considering using an IUD/IUS following a TOP. A study of ongoing contraception post-TOP from our service in 2008 showed that $9.5 \%$ of women had an IUD/IUS inserted immediately following a TOP. ${ }^{10}$ It is possible that this apparent increase in 'interest' in intrauterine contraception in our current study may reflect the impact of a Scottish Government sexual health strategy that used social marketing to promote awareness of the most effective long-acting methods of contraception. ${ }^{20}$ Our surveys were anonymous, so it is unlikely that women felt compelled to indicate interest in this method of contraception. It does, however, suggest that the consultation prior to a TOP is a good opportunity for health professionals to provide accurate information to women about the IUD/IUS, since motivation to use this method may be high and its uptake may protect women from a subsequent TOP. Although clearly our study was limited by sample size, the findings do add weight to the importance of abortion care providers being trained and funded to be able to provide the IUD/IUS to women at the time of abortion, if they wish this and if it is appropriate to do so. ${ }^{10}$
Author affiliations

${ }^{1}$ Clinical Research Fellow, Department of

Reproductive and Developmental Sciences, University of Edinburgh, Edinburgh and Chalmers Sexual and

Reproductive Health Centre, Edinburgh, UK

${ }^{2}$ Consultant in Gynaecology and Reproductive

Health, Department of Reproductive and

Developmental Sciences, University of Edinburgh,

Edinburgh and Chalmers Sexual and Reproductive

Health Centre, Edinburgh, UK

${ }^{3}$ Honorary Professor of Sexual and Reproductive

Health, Department of Reproductive and

Developmental Sciences, University of Edinburgh,

Edinburgh, UK

${ }^{4}$ Professor of Sexual and Reproductive Health

Research, London School of Hygiene and Tropical

Medicine, London, UK

${ }^{5}$ Medical Student, College of Medicine and Veterinary

Medicine, University of Edinburgh, Edinburgh, UK

Acknowledgements The authors are grateful to clinical research nurses Helen Dewart and Anne Johnstone for assistance with conduct of the survey.

Funding This project was undertaken with funding from Scottish Government, Public Health Division as part of a series of projects aimed at improving the patient journey through abortion services.

Competing interests None.

Provenance and peer review Not commissioned; externally peer reviewed.

\section{REFERENCES}

1 United Nations, Department of Economic and Social Affairs, Population Division. World Contraceptive Use. 2009. http:// www.un.org/esa/populations/publications/contraceptive2009 [accessed 2 April 2012].

2 D'Arcangues C. Worldwide use of intrauterine devices for contraception. Contraception 2007;75:S2-S7.

3 Lader D. Contraception and Sexual Health (2008/09). Opinion Survey Report No. 41. Office for National Statistics. http:// www.statistics.gov.uk [accessed 28 August 2012].

4 Mansour D, Inki P, Gemzell-Danielsson K. Efficacy of contraceptive methods: a review of the literature. Eur J Contracept Reprod Health Care 2010;15:4-16.

5 Information Services Division. Abortion Statistics 2011. May 2012. http://www.isdscotland.org/Health-Topics/Sexual-Health/ Publications/2012-05-29/2012-05-29-Abortions-Summary2011.pdf [accessed 28 August 2012].

6 Department of Health. Abortion Statistics England and Wales 2011. May 2012. https://www.wp.dh.gov.uk/transparency/files/ 2012/05/Commentary1.pdf [accessed 28 August 2012].

7 National Institute for Health and Clinical Excellence. LongActing Reversible Methods of Contraception. 2005. http://www. nice.org.uk/CG30fullguideline [accessed 28 August 2012].

8 Goodman S, Hendlish SK, Reeves MF, et al. Impact of immediate postabortal insertion of an intrauterine contraception on repeat abortion. Contraception 2008;78:143-148.

9 Heikinheimo O, Gissler M, Suhonen S. Age, parity, history of repeat abortion and contraceptive choices affect the risk of repeat abortion. Contraception 2008;78:149-154. 
10 Cameron ST, Glasier A, Chen ZE, et al. Effect of contraception provided at termination of pregnancy and incidence of subsequent termination of pregnancy. Br J Obstet Gynaecol 2012;119:1074-1080.

11 Asker C, Stokes-Lampard H, Beaven J, et al. What is it about intrauterine devices that women find unacceptable? Factors that make women non-users: a qualitative study. J Fam Plann Reprod Health Care 2006;32:89-94.

12 Glasier A, Scorer J, Bigrigg A. Attitudes of women in Scotland to contraception: a qualitative study to explore the acceptability of long-acting methods. J Fam Plann Reprod Health Care 2008;34:213-217.

13 French RS, Mercer CH, Kane R, et al. What impact has England's Teenage Pregnancy Strategy had on young people's knowledge of and access to contraceptive services? J Adolesc Health 2007;41:594-601.

14 McLoone P. Carstairs scores for Scottish postcode sectors from the 2001 census - report. 2000. http://www.sphsu.mrc. ac.uk/library/otherreports/Carstairs_report [accessed 12 July 2012].

15 Powell-Jackson R, Glasier A, Cameron ST. Benefits of using a digital video disk for providing information about abortion to women requesting termination of pregnancy. Contraception 2010;81:537-541.

16 Whitaker AK, Johnson LM, Harwood B, et al. Adolescent and young adult women's knowledge and attitudes toward the intrauterine device. Contraception 2008;78:

211-217.

17 Whitaker AK, Terplan M, Gold M, et al. Effect of a brief educational intervention on the attitudes of young women toward the intrauterine device. J Pediatr Adolesc Gynecology 2010;23:116-120.

18 Fleming KL, Sokoloff A, Raine TR. Attitudes and beliefs about the intrauterine device among teenagers and young women. Contraception 2010;82:178-182.

19 Faculty of Sexual \& Reproductive Healthcare Clinical Effectiveness Unit. Intrauterine Contraception. 2007. http:// www.fsrh.org/pdfs/CEUGuidanceIntrauterineContraception Nov07.pdf [accessed 26 July 2012].

20 NHS Health Scotland. Awareness and Knowledge of Long Acting Reversible Contraception: What Women and Professionals in Scotland Need. 2009. http://www.askforlarc. nhs.uk/_assets/docs/LARC_Awareness_Scottish_Report_2009. pdf [accessed 27 August 2012]. 